\title{
A structural comparison of human serum transferrin and human lactoferrin
}

\author{
Jeremy Wally $\cdot$ Susan K. Buchanan
}

Received: 25 August 2006/ Accepted: 28 November 2006/ Published online: 11 January 2007

(C) Springer Science+Business Media B.V. 2007

\begin{abstract}
The transferrins are a family of proteins that bind free iron in the blood and bodily fluids. Serum transferrins function to deliver iron to cells via a receptor-mediated endocytotic process as well as to remove toxic free iron from the blood and to provide an antibacterial, low-iron environment. Lactoferrins (found in bodily secretions such as milk) are only known to have an anti-bacterial function, via their ability to tightly bind free iron even at low $\mathrm{pH}$, and have no known transport function. Though these proteins keep the level of free iron low, pathogenic bacteria are able to thrive by obtaining iron from their host via expression of outer membrane proteins that can bind to and remove iron from host proteins, including both serum transferrin and lactoferrin. Furthermore, even though human serum transferrin and lactoferrin are quite similar in sequence and structure, and coordinate iron in the same manner, they differ in their affinities for iron as well as their receptor binding properties: the human transferrin receptor only binds serum transferrin, and two distinct bacterial transport systems are used to capture iron from serum transferrin and lactoferrin.
\end{abstract}

J. Wally · S. K. Buchanan ( ()

National Institute of Diabetes and Digestive and Kidney Diseases, National Institutes of Health, 50 South Drive, Bethesda, MD 20892, USA

e-mail: skbuchan@helix.nih.gov
Comparison of the recently solved crystal structure of iron-free human serum transferrin to that of human lactoferrin provides insight into these differences.

Keywords Transferrin - Lactoferrin · Iron transport · Human transferrin receptor - Bacterial transferrin receptor

\section{Introduction}

Control of the level of free iron in the body is critical to protect the body from the deleterious effects of having free iron in the aerobic environment of the blood and bodily fluids. Damage occurs through the formation of harmful free radicals as a product of the conversion of ferrous iron to ferric iron via the Fenton reaction, and from the insolubility of the resultant ferric iron (Wandersman and Delepelaire 2004). Iron levels are controlled via a family of proteins known as transferrins (Aisen et al. 1978; Harris and Aisen 1989). Transferrins exist in the blood (serum transferrins or TFs), in other bodily secretions (lactoferrins or LTFs) and in avian egg white (ovotransferrins or oTFs). A fourth class of transferrins, melanotrasferrins, was first identified in human skin cancer cells (Woodbury et al. 1980) but now is known to be expressed across a broad range of tissue types, and is of unknown function. In addition to a simple 
protective role, TFs transport iron in the blood by picking up free ferric iron and delivering it to cells in a receptor-mediated endocytotic process, in which the TF-receptor complex is internalized, iron is released in the endosome, and the complex is recycled to the cell surface where the TF is released (Harris and Aisen 1989). A cryo-electron microscopy model of human TF (hTF) bound to the human transferrin receptor (TFR; Fig. 1)
(Cheng et al. 2004) as well as analyses of the residues involved in binding (Giannetti et al. 2003, 2005; Liu et al. 2003; Teh et al. 2005; Xu et al. 2005) suggests that a conformational change in hTF and/or the TFR occurs upon receptor binding. Interestingly, no iron transport role for LTFs and oTFs is known, and neither human LTF nor chicken oTF binds to the TFR. Instead, the major function of LTFs and oTFs (and a secondary

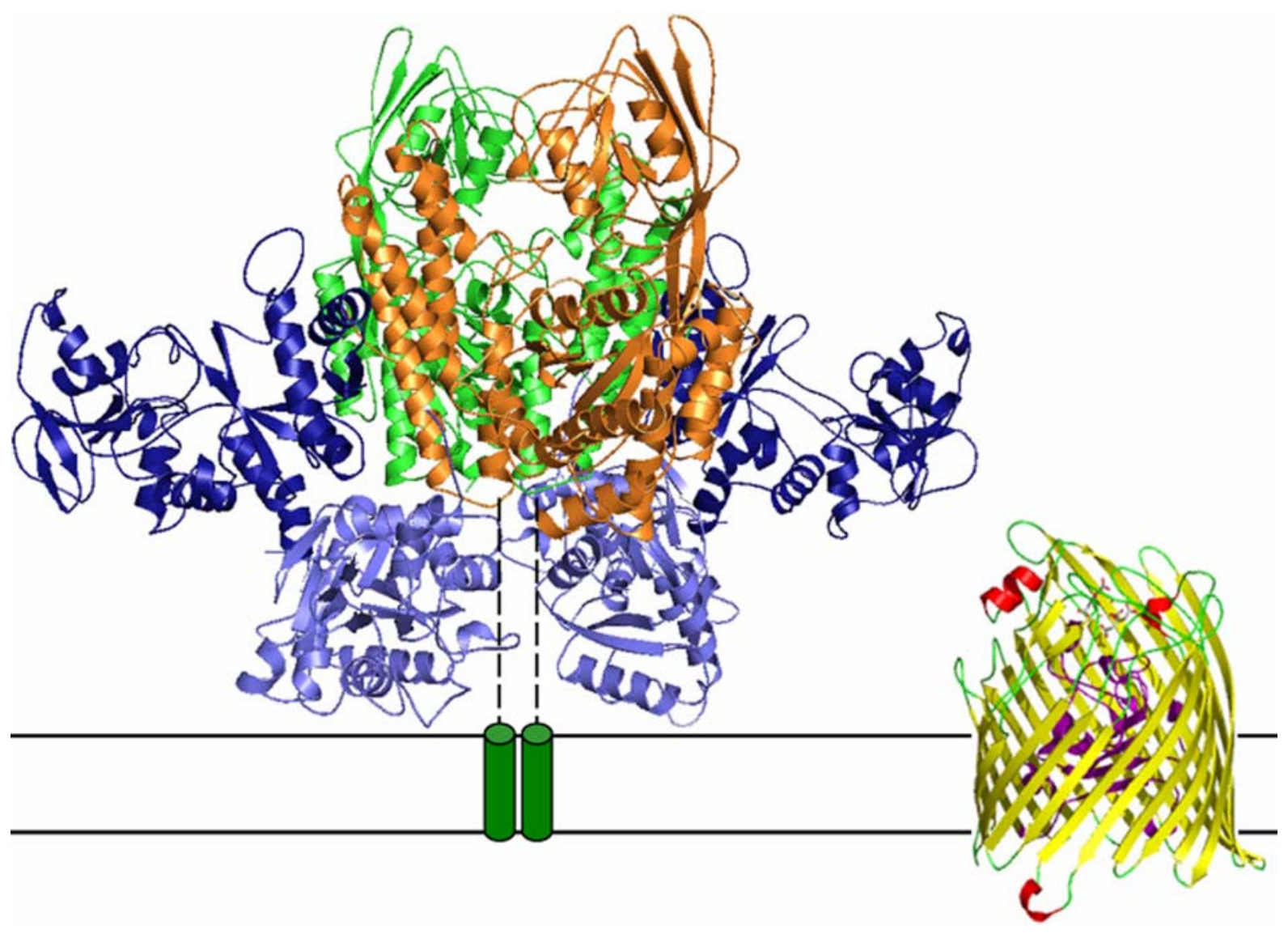

Fig. 1 Structural model of iron-free hTF bound to the ectodomain of the TFR and the crystal structure of a 22stranded $\beta$-barrel bacterial iron transporter. Left The model of the TFR dimer (one monomer green and one orange) (Lawrence et al. 1999) bound to two molecules of iron-free hTF (Wally et al. 2006) was built using the cryoelectron microscopy structural model of holo-hTF bound to the TFR (Cheng et al. 2004). The iron-free hTF Nlobes (light blue) and C-lobes (dark blue) were placed in corresponding positions to that of the iron-bound lobes using SPDBV (Guex and Peitsch 1997), which is supported by recent low-resolution data (Cheng et al. 2005). The two lobes of each hTF molecule are separated by ( $9 \AA$ from each other as compared to their positioning in the apo-hTF structure. The missing protease-like domain (dotted lines) and transmembrane helices (green cylinders) of the TFR are shown. Right The crystal structure of FecA from Escherichia coli (Yue et al. 2003) is shown with the barrel domain colored by secondary structure ( $\beta$-strands yellow and helices red) and the plug domain colored in purple. The bound siderophore (diferric dicitrate) is shown in stick representation. Bacterial transferrin and lactoferrin receptors are thought to have the same basic architecture as FecA (see Fig. 6). The two structures are shown to scale 
function of TFs) is thought to be keeping the iron concentration low in bodily fluids to prevent invading bacteria from acquiring iron. However, some bacteria can circumvent the resultant iron-deficient environment created by transferrins: many pathogenic bacteria have evolved systems to acquire iron from host proteins, including both TF and LTF, by expressing outer membrane proteins that can bind and remove the iron (Fig. 1).

The crystal structures of transferrin family members across all species that have been solved to date are quite similar, which is not surprising since TFs, LTFs and oTFs share 60-80\% sequence identity (Anderson et al. 1989; Kurokawa et al. 1995, 1999; Rawas et al. 1996, 1997; Moore et al. 1997; Jeffrey et al. 1998; Jameson et al. 1999; Khan et al. 2001; Hall et al. 2002; Kumar et al. 2002; Guha Thakurta et al. 2003; Thakurta et al. 2004; Wally et al. 2006). All structures consist of two homologous lobes (termed the $\mathrm{N}$ - and C-lobes) connected by a short peptide linker, which is helical in LTFs and unstructured in TFs and oTFs. Each lobe can be further divided into two subdomains (N1 and N2 in the $\mathrm{N}$-lobe and $\mathrm{C} 1$ and $\mathrm{C} 2$ in the $\mathrm{C}$-lobe) that form a cleft inside of which the iron is bound. The iron-coordinating residues in the $\mathrm{N}$ - and C-lobes of transferrin family members are strictly conserved, consisting of an aspartic acid, two tyrosines and a histidine, as well as an arginine that coordinates a requisite anion. Based upon comparisons of transferrins whose iron-bound and iron-free structures are known, it is thought that in each lobe, the subdomains move as rigid bodies around a few hinge residues that lie between the subdomains, opening and twisting as iron is released and closing as iron is bound (Anderson et al. 1989; Kurokawa et al. 1995, 1999; Jeffrey et al. 1998; MacGillivray et al. 1998; Jameson et al. 1999; Guha Thakurta et al. 2003; Thakurta et al. 2004).

Humans produce both serum transferrin (hTF) and LTF, and though these proteins are similar in sequence (61.4\% identical; Fig. 2) and structure (Fig. 3), they function quite differently, as described above. In addition to having a role in iron transport through its interactions with the TFR, hTF releases iron at a higher $\mathrm{pH}$ (around $\mathrm{pH}$
5.5) than does human LTF (around $\mathrm{pH} 3$ ) which likely relates to the need for hTF to release iron after endocytosis of the hTF-TFR complex into the endosome (Mazurier and Spik 1980; Baker and Baker 2004) and the need for LTF to hold onto to iron in low $\mathrm{pH}$ environments such as the mucus layer of the stomach. This $\mathrm{pH}$-dependent iron release by hTF is thought to relate to the presence of a dilysine trigger in the $\mathrm{N}$-lobe (He et al. 1999), in which two lysine residues extend from the N1 and N2 subdomains and interact with each other in iron-bound hTF (MacGillivray et al. 1998). It is thought that in an acidic environment, the sidechains of these lysines would become charged, repelling each other and forcing the $\mathrm{N}$-lobe open, releasing the iron. In the hTF C-lobe, however, the dilysine trigger is replaced by a triad of residues, which is thought to contribute to the slower release of iron from the C-lobe as compared to the N-lobe (Halbrooks et al. 2003). Human LTF contains neither the $\mathrm{N}$-lobe dilysine trigger nor the C-lobe triad. However, structural differences between hTF and LTF are also likely to be important, since mutations of these residues fail to explain the differences in $\mathrm{pH}$-dependent release of iron (Nurizzo et al. 2001; Baker and Baker 2004) and LTFs from other species contain the dilysine trigger (Peterson et al. 2002).

Pathogenic bacteria make separate transport systems to acquire iron from hTF and human LTF, consisting of an integral outer membrane receptor/transporter (TbpA, LbpA), a lipid anchored co-receptor (TbpB, LbpB) on the cell surface, and several other proteins in the periplasm and inner membrane. The TbpA/TbpB transport system can only acquire iron from human transferrin, whereas the LbpA/LbpB system can only use iron from lactoferrin. This need for bacteria to synthesize and express two distinct transport systems indicates that either the structures of hTF and human LTF are sufficiently different, and/or the requirements for removing iron from hTF and LTF by the bacterial receptors differ. We have recently solved the crystal structure of iron-free hTF (Wally et al. 2006). Comparison of it to the structure of human LTF gives some insight into the functional differences between these proteins and their receptor binding properties. 


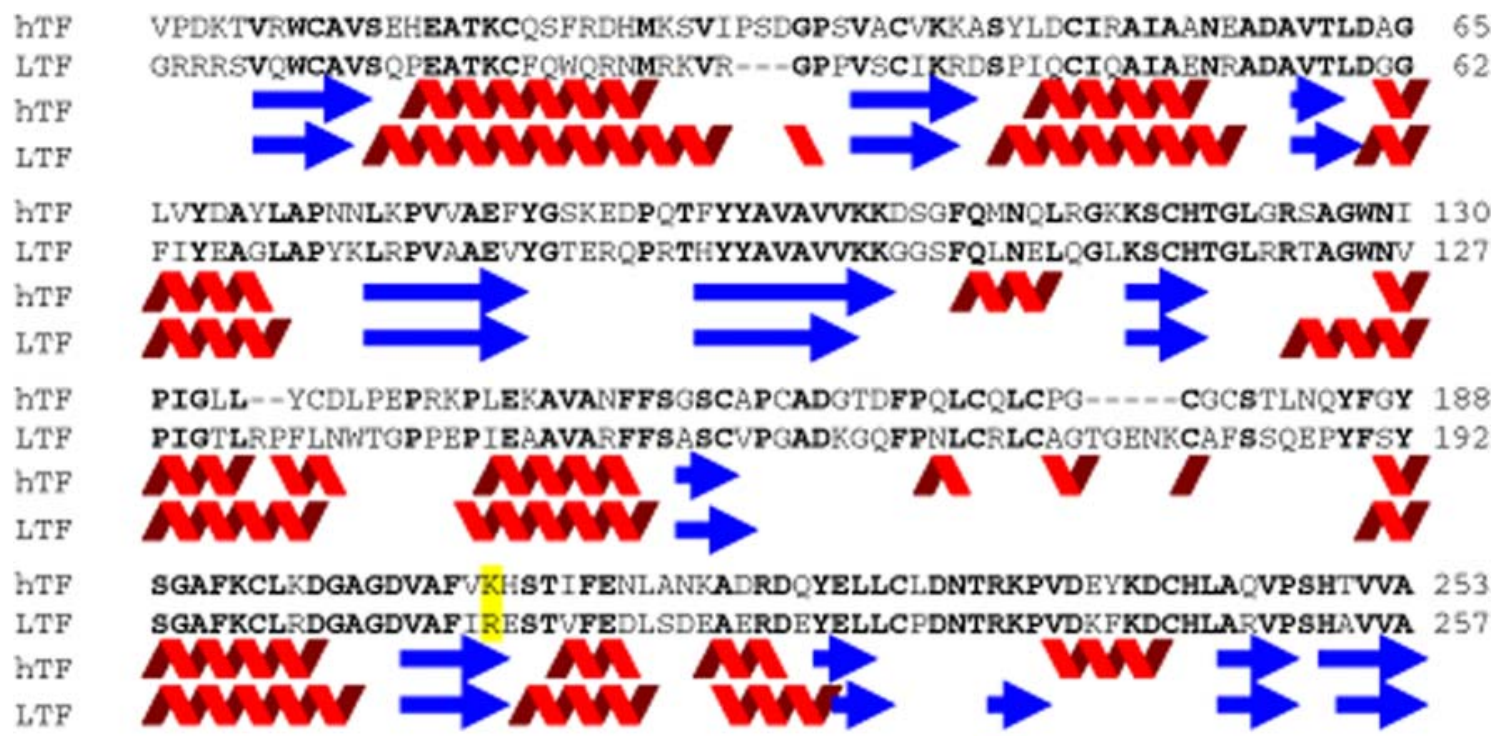

hTF RSMGGKEDLIWELLNOAOEHFGKDKSKEFOLFSSPHG-KDLLFKDSAHGFLKVPPRMDAKMYLGY 317 LTF RSVNGKEDAIWNLLRQAQEKFGKDKSPKFQLFGSPSGQKDLLFKDSAIGSSRVPPRIDSGLYLGS 322
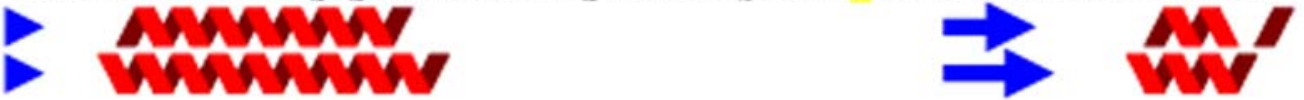

EYVTAIRNLREGTCPEAPTDECKPVKWCALSHHERLKCDEWSVNSVGKIECVSAETTEDCIAKIM 382 GYFTAIQNLRK--SEEEVAARRARVVWCAVGEQELRKCNQWSGLSEGSVTCSSASTTEDCIA--L 383 infin
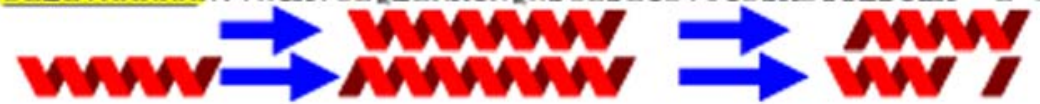

NGEADAMSLDGGFVYIAGKCGLVPVLAENYNK---SD---NCEDTPEAGYFAVAVVKKSASDLTW 441 KGEADAMSLDGGYVYTAGKCGLVPVLAENYKSQQSSDPDPNCVDRPVEGYLAVAVVRRSDTSLTW 450

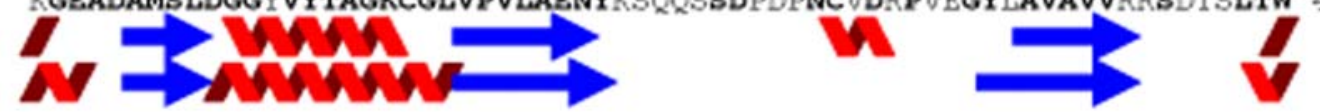

DNLKGKKSCHTAVGRTAGWNIPMGLLYNKINICRFDEFFSEGCAPGSKKDSSLCKLCMG--SGLN 504 NSVKGKKSCHTAVDRTAGWNIPMGLLFNQTGSCKFDEYFSQSCAPGSDPRSNLCALCIGDEQGEN 515
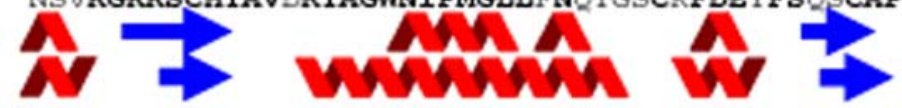

LCEPNNKEGYYGYTGAFRCLVE-KGDVAFVKHQTVPQNTGGKNPDPWAKNLNEKDYELLCLDGTR 568 KCVPNSNERYYGYTGAFRCLAENAGDVAFVKDVTVLQNTDGNNNDAWAKDLKLADFALLCLDGKR 580

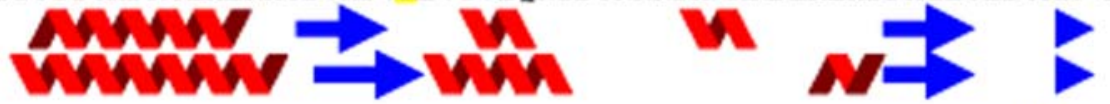

KPVEEYANCHLARAPNHAVVTRKDKEACVHKILRQQQHLFGSNVTDCSGNFCLFRSETKDLLFRD 633 KPVTEARSCHLAMAPNHAVVSRMDKVELKQVLLHQQAKFGRNGSDCPDKFCLFQSETKNLLFND 645

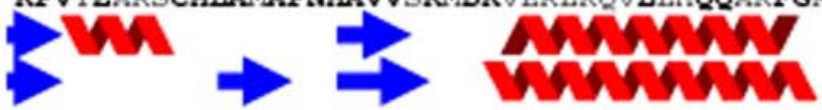

DTVCLAKLHDRNTYEKYLGEEYVKAVGNLRKCSTSSLLEACTFRRP 679

NTECLARLHGKTTYEKYLGPOYVAGITNLKKCSTSPLLEACEFLRK 691
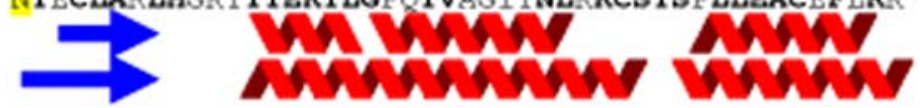
4 Fig. 2 Sequence alignment of hTF and LTF created using the program ClustalW (http://www.ebi.ac.uk/clustalw) (Thompson et al. 1994). Identical residues are shown in bold. The dilysine trigger residues (Lys206 and Lys296) and triad residues (Lys534, Arg632 and Asp634) of hTF and highlighted in yellow as are the corresponding residues in LTF. The linker regions between the lobes of both proteins are highlighted in yellow and underlined. Under the sequences are given their secondary structures taken from the protein databank files $2 \mathrm{HAV}$ for hTF (Wally et al. 2006) and 1CB6 for LTF (Jameson et al. 1999). Strands are shown as blue arrows and helices as red ribbons

\section{Structural comparison of human serum transferrin and human lactoferrin}

Like all other TFs, hTF and LTF have the same basic architecture, with two similar lobes attached by a linker region and each lobe having two subdomains that form a deep cleft in which iron is bound. One of the most striking differences between the structures is that in LTF the linker is helical and in hTF the corresponding residues are in an extended (unstructured) conformation (Fig. 3). It had been proposed that the helical nature of the linker in LTF is

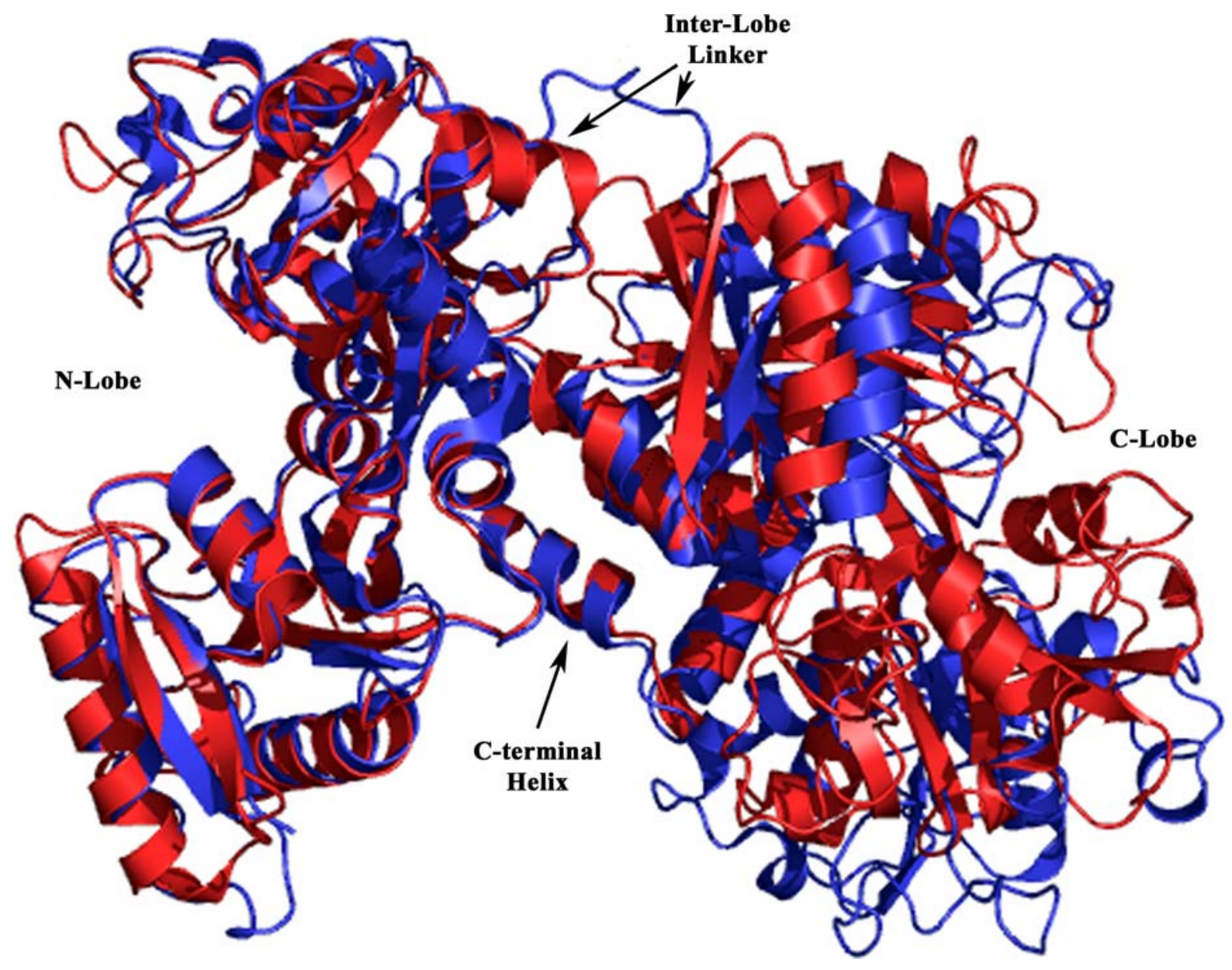

Fig. 3 Structural superposition of hTF (blue) with LTF (red) created by aligning and superimposing the N1 subdomains of the two proteins. Though the N-lobes of both proteins superimpose quite well, the C-lobe of LTF is rotated away from its $\mathrm{N}$-lobe as compared to the C-lobe of
hTF, and is less open than the C-lobe of hTF. The Cterminal helices of both proteins are positioned in a very similar location, however. The superpositions were performed using SPDBV (Guex and Peitsch 1997) and the figure was made using PyMOL (DeLano 2002) 
solely responsible for the iron-binding cooperativity observed between the $\mathrm{N}$ - and C-lobes (Day et al. 1992; Ward et al. 1996; Baker and Baker 2004), but several studies have also demonstrated cooperativity between the N-and C-lobes of hTF and of oTF (Lin et al. 1991, 1994; Kurokawa et al. 1994; Beatty et al. 1996; Gumerov et al. 2003; Okamoto et al. 2004). It is possible that having a helical linker simply modulates this cooperativity in LTF, by providing a more rigid link between the two lobes (Baker and Baker 2004). Interestingly, a salt bridge is found between Arg341 within the helical linker of LTF and Asp390 in the C1 subdomain (Fig. 4), supporting a more connected interaction between the lobes of LTF. No side chain interactions between the unstructured linker of hTF and either of its lobes are observed, and the linker in hTF lies further away from the rest of the protein as compared to the linker in LTF.

Another interesting difference between hTF and LTF, with regard to their inter-lobe linkers, is



Fig. 4 Comparison of the inter-lobe interactions in hTF (blue, with the $\mathrm{N}$-lobe colored lighter and the C-lobe darker) and LTF (red, with the N-lobe colored lighter and the C-lobe darker). In hTF, an inter-lobe salt bridge is found between Arg308 on the loop between sheet $\mathrm{k}$ and helix 10 in the N1 subdomain and Asp376 in helix 2 of the C1 subdomain. A similar salt bridge is observed for LTF, between Asp315 and Lys386. hTF has an additional inter- that the linker in hTF is bounded by two cysteine residues (amino acids 331 and 339) that form disulfide bonds between the N1 and N2 subdomains (cysteines 331-137) and between the $\mathrm{C} 1$ and C2 subdomains (cysteines 339-596). These disulfide bonds are not present in LTF (Fig. 5), and furthermore there is no disulfide bonding between the subdomains in either lobe of LTF. These disulfide bonds in hTF each act to tether the linker to the $\mathrm{N} 2$ and $\mathrm{C} 1$ subdomains, respectively, with the linker extending away from the lobes of hTF. This arrangement might give the lobes of hTF the ability to change their relative orientations while maintaining a limit to the change (the length of the linker) via these strong, anchoring disulfide bonds. The helical linker in LTF, with its salt bridge to the $\mathrm{C} 1$ subdomain, would likely prohibit this sort of change, limiting the degree of relative lobe movement. A third disulfide bond is also present in hTF and not in LTF (between cysteines 161 and 179 in the N2 subdomain), though this bond occurs between two loop regions.

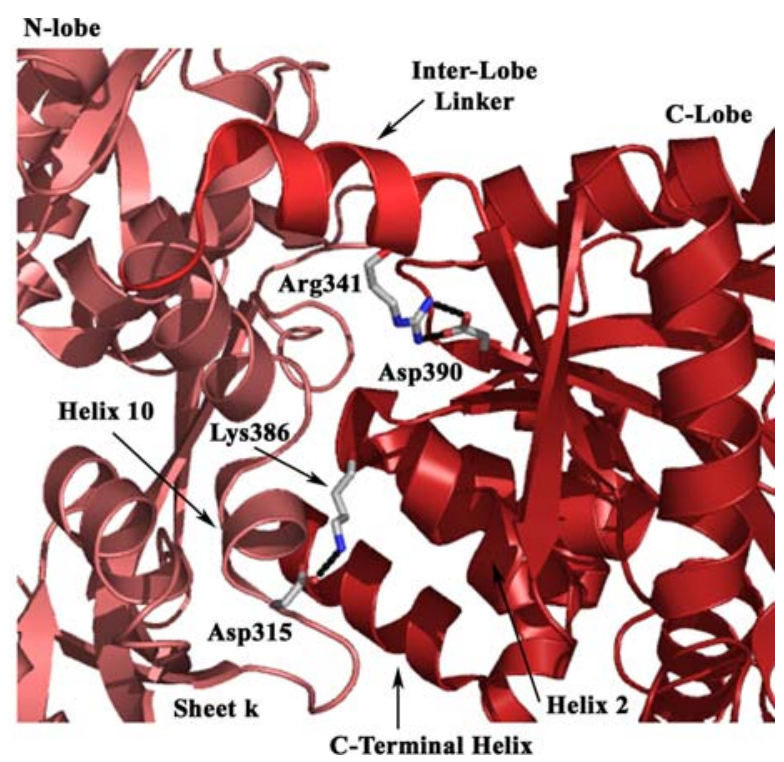

lobe salt bridge between Asp240 in the N2 subdomain and Arg678 in the $\mathrm{C} 1$ subdomain that is not observed in LTF. Arg678 in hTF caps the C-terminal helix that lies between the lobes. LTF also has a salt bridge between Arg341 within the helical inter-lobe linker and Asp390 in the C1 subdomain. The figure was made using PyMOL (DeLano 2002) 


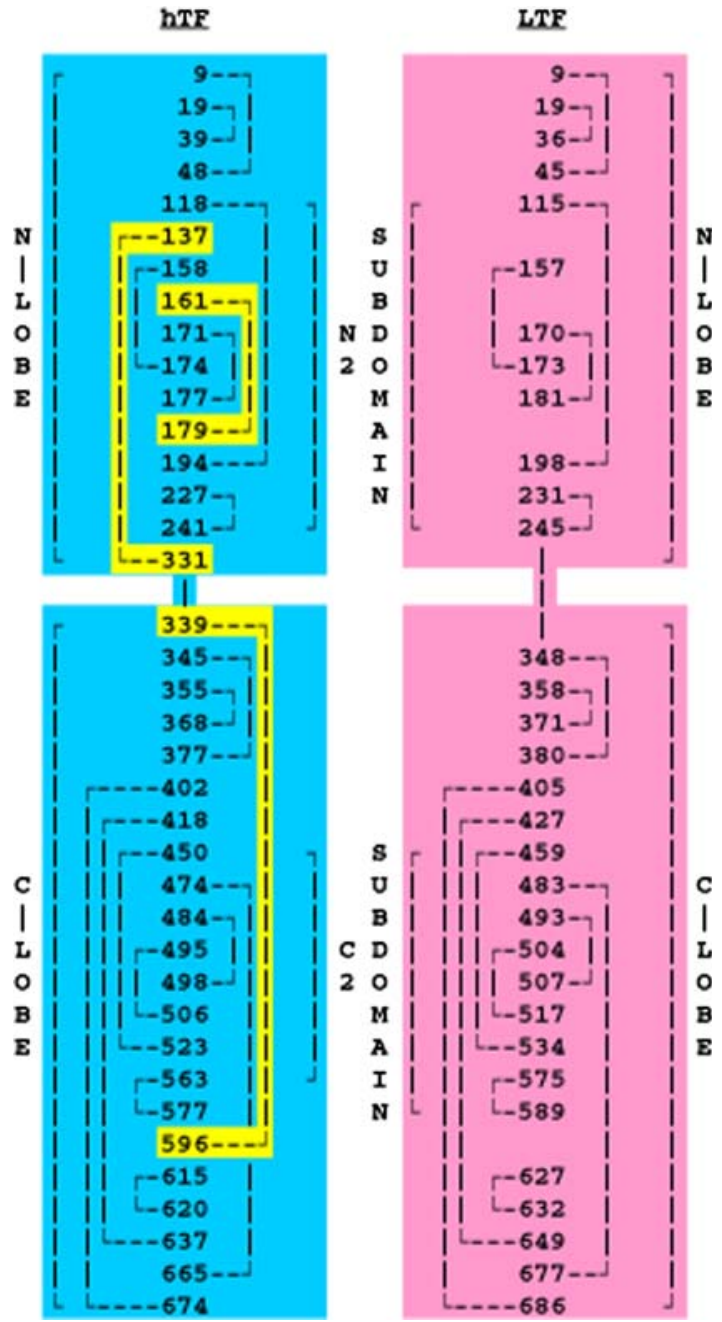

Fig. 5 Pattern of disulfide bonding within the structure of hTF (2HAV; blue) (Wally et al. 2006) as compared to that of LTF (1CB6; red) (Jameson et al. 1999). The residues are aligned according to Fig. 1. hTF contains three additional disulfide bonds as compared to LTF, between cysteines 137 and 331, 161-179 and 339 and 596 (highlighted in yellow)

The difference in the $\mathrm{pH}$-dependent iron release for the $\mathrm{N}$ - and $\mathrm{C}$-lobes of hTF has been postulated to arise from the dilysine trigger in the $\mathrm{N}$-lobe and the triad of charged residues in the C-lobe. However, the structure of iron-free hTF revealed another striking distinction between the two lobes of hTF (Baker and Baker 2004; Wally et al. 2006). The N- and C-lobes of hTF differ in the secondary structure of the regions surrounding the hinge residues: in the N-lobe the hinge residues lie adjacent to a 2 -stranded $\beta$-sheet as found in other transferrin family members, including LTF. In the C-lobe of hTF this region is unstructured, whereas the C-lobe of LTF has a 2 -stranded $\beta$-sheet analogous to the hTF and LTF $\mathrm{N}$-lobe structures. This structural difference between the hTF $\mathrm{N}$ - and C-lobes may contribute to their individual iron binding properties via a difference in the rigidity of the lobes; likewise, this structural difference between the C-lobes of hTF and LTF may play a role in the distinct iron affinities of the two molecules.

Other than the linker between the lobes, another important consideration is the nature of the inter-lobe contact. All TFs have a relatively small amount of buried surface area between the lobes, usually around 400-600 $\AA^{2}$. Inter-lobe contacts are strengthened in iron-free hTF by two additional salt bridges, formed between Arg308 in the N1 subdomain and Asp376 in the C1 subdomain, and between Asp240 in the N2 subdomain and Arg678 in the $\mathrm{C} 1$ domain (Fig. 4) (Wally et al. 2006). Arg678 is the penultimate residue in hTF and lies at the end of the C-terminal helix, which is positioned between the $\mathrm{N}$ - and $\mathrm{C}$-lobes. Interestingly, only a single inter-lobe salt bridge is observed between the lobes of LTF, in both the iron-bound and ironfree forms, between Asp315 in the N2 subdomain and Lys386 in the $\mathrm{C} 1$ subdomain. This salt bridge makes a similar connection as seen in hTF (Arg308-Asp376), linking helix 2 of the C-lobe with the loop between $\beta$-sheet $\mathrm{k}$ and helix 10 of the N-lobe, though at different locations on the loop and helix 2 (secondary structural designations as in Wally et al. 2006). No salt bridges are observed between the N-lobe and the C-terminal helix of LTF. These differences in inter-lobe contacts may restrict mobility of the $\mathrm{N}$ - and C-lobes, possibly influencing iron affinities and receptor (both human and bacterial) recognition.

Comparison of the tertiary structure of ironfree hTF to that of iron-bound rabbit TF suggested that as the lobes of hTF open and close, the orientation of the lobes relative to one another does not change. The $\mathrm{N} 1$ and $\mathrm{C} 1$ subdomains of hTF superimpose exactly on the N1 and C1 subdomains of rabbit TF (Wally et al. 2006). However, comparison of the tertiary structure of 
iron-free hTF to that of iron-bound human LTF indicates that the overall orientation of the lobes of these two molecules is different (Fig. 3). When aligning the N1 subdomain of hTF on the N1 subdomain of LTF, the C1 subdomains of hTF and LTF do not superimpose. The C-lobe of LTF is rotated toward the N-lobe by about $8.5^{\circ}$ as compared to the C-lobe of hTF, though the position of the C-terminal helix in both molecules is identical. This tertiary structural difference between hTF and LTF is likely due to the helical inter-lobe linker in LTF, which is shorter than the unstructured inter-lobe linker in hTF and more closely associated with the lobes of LTF, as well as the differences in inter-lobe interactions already described.

\section{Transferrin receptor binding}

Serum transferrin functions to transport iron in the bloodstream to cells and deliver the iron via interaction with the TFR. After binding, the hTFTFR complex is internalized and exposed to the low $\mathrm{pH}$ environment of the endosome. At this acidic $\mathrm{pH}$, hTF releases its iron which is then transported into the cell via Nramp2 (Fleming et al. 1998). The entire hTF-TFR complex is then recycled to the cell surface, where at neutral $\mathrm{pH}$, the affinity of the TFR for iron-free hTF is low, so the hTF is released. Recent cryo-electron microscopic models of both iron-free and iron-bound hTF bound to a soluble form of the TFR (Cheng et al. 2004, 2005), as well as many studies of receptor binding have shed light on the nature of the interaction between hTF and the TFR (Fig. 1). In the iron-bound hTF-TFR cryo-electron microscopy model, the dimeric TFR interacts with two molecules of hTF, with interactions made between the $\mathrm{C} 1$ subdomain and the $\mathrm{N}$-lobe of hTF. The C-lobe sits above the N-lobe, and the $\mathrm{N}$-lobe is close to the membrane. However, fitting of the lobes of hTF (the authors used the known human iron-bound N-lobe structure and the rabbit iron-bound $\mathrm{C}$-lobe structure) in the electron density necessitated a $9 \AA$ separation of the two lobes relative to one another in the full-length hTF structure. This feature, along with some conflicting evidence about which residues interact with the TFR (Giannetti et al. 2003, 2005; Liu et al. 2003; Teh et al. 2005; Xu et al. 2005), has led to the suggestion that conformational changes must take place in hTF and/or the TFR upon complex formation, which could not be seen within the resolution of the electron density maps (Wally et al. 2006).

The ability of hTF to undergo a conformational change upon binding to the TFR might be a unique requirement for binding. It is possible that the inability of LTF to undergo a similar conformational change might explain its inability to bind to the TFR, since analysis of the residues that might interact with the TFR in hTF and LTF does not suggest any obvious differences that account for the lack of TFR binding by LTF (Wally et al. 2006), and analyses of sequence differences in the context of tertiary structure fail to reveal any obvious differences between hTF and LTF. Furthermore, the structures of the $\mathrm{N}$ - and C-lobes of hTF and LTF overall are very similar (for the iron-free structures, the $\mathrm{C} \alpha$ r.m.s.d. is $0.91 \AA$ for the N-lobe, $0.83 \AA$ for the $\mathrm{C} 1$ subdomain and $0.69 \AA$ for the $\mathrm{C} 2$ subdomain). However, comparison of the two structures (Fig. 3) suggests that the relative orientations of the lobes in hTF and LTF might be important, in that it would position these residues in different relative locations in LTF as compared to hTF, which might be incompatible with TFR binding. Details of the amino acids of hTF that interact with the TFR are currently limited by the lack of a high-resolution structure of the hTF-TFR complex.

The interactions between the two lobes of hTF are likely critical in allowing the conformational change that is suggested to occur in hTF upon binding to the TFR. Therefore differences in these interactions between hTF and LTF might explain the lack of binding of LTF to the TFR and the lack of iron delivery to cells by LTF. Both hTF and LTF have a relatively small area of hydrophobic contact between their lobes (for the iron-free proteins, $552 \AA^{2}$ for hTF and $660 \AA^{2}$ for LTF) and contain a similarly located salt bridge that links the $\mathrm{N} 1$ and $\mathrm{C} 1$ subdomains in each protein. However, for hTF the linker is unstructured and extended, and is bounded by two inter-subdomain disulfide bonds. For LTF, the linker is helical and more closely associated with 
the lobes, and has a salt bridge to the $\mathrm{C} 1$ subdomain. In addition, hTF has an additional salt bridge that connects the $\mathrm{N} 2$ subdomain to the C1 subdomain via the C-terminal helix, that is absent in LTF. Taken together, this analysis suggests that the inter-lobe interactions in LTF may be tighter as compared to hTF, with hTF having more flexibility between its lobes as facilitated by its flexible linker and stronger connection between its C-terminal helix and $\mathrm{N}$-lobe. This helix has previously been suggested to be important for inter-lobe communication (Jameson et al. 1999), and could insert further between the lobes during a conformational change. Furthermore, this differential interaction between the lobes of hTF and LTF might be related to their differential iron affinities, by affecting the cooperativity of iron binding between the lobes, as has also been previously suggested (Baker and Baker 2004). Further analyses of these salt bridging and disulfide bonding residues are warranted.

\section{Bacterial transferrin and lactoferrin receptors}

Pathogenic bacteria express outer membrane protein complexes that allow them to interact with host iron-containing proteins and to extract the iron from these proteins. In addition to expressing transport systems for both $\mathrm{TF}$ and LTF, transporters for other host proteins, including hemoglobin, have been identified (Rohde and Dyer 2003). The TF and LTF outer membrane receptors are predicted to be 22 -stranded $\beta$-barrel type integral outer membrane proteins of (100 kDa, having N-terminal plug domains which are thought to lie within the barrel as in other TonB-dependent outer membrane transporters (Boulton et al. 2000; Oakhill et al. 2005) based upon their sequence homology to other proteins of this type (Wiener 2005) (Fig. 1). The outer membrane receptor (TbpA or LbpA) is thought to bind to a host protein (transferrin or lactoferrin), extract the iron, and transport it into the periplasm, explaining the observation that neisserial mutants lacking TbpA are unable to internalize iron from TF (Cornelissen et al. 1992). The energy for iron transport (and possibly iron removal from transferin or lactoferrin) is thought to be derived from the TonB-ExbBExbD complex that resides in the bacterial inner membrane, since TbpA and LbpA both contain a specific amino acid sequence located near their $\mathrm{N}$-terminus (the TonB box) that has been identified in all other integral outer membrane transport proteins. TonB is thought to have an extended structure that spans the periplasm, allowing interaction of its C-terminal domain with the TonB box (Pawelek et al. 2006; Shultis et al. 2006). TbpA and LbpA each function with a membrane-associated accessory protein ( $\mathrm{TbpB}$ or transferrin binding protein $\mathrm{B}$ and $\mathrm{LbpB}$ or lactoferrin binding protein $\mathrm{B}$ ) of $65-85 \mathrm{kDa}$ based upon strain, and is thought to assist in distinguishing iron-bound from iron-free targets, since neisserial mutants lacking $\mathrm{TbpB}$ can still internalize iron from $\mathrm{TF}$, though at a reduced level (Anderson et al. 1994). However, the exact stoichiometry of the TbpA-TbpB and LbpALbpB complexes and the nature of the association between the complex components before binding TF or LTF are currently unknown. Several studies have suggested that the ratio is $2: 1$ for TbpA to TbpB, suggesting that that each TbpATbpB complex binds with the ability to extract iron from both lobes of TF (Boulton et al. 1997, 1998). However, lack of structural data on any of the bacterial components of these complexes limits our understanding of the process.

A model of hTF binding and iron removal by the TbpA-TbpB complex exists based upon substantial functional and biochemical evidence (Boulton et al. 1999; Evans and Oakhill 2002). In the model, TbpA and TbpB reside at the bacterial outer membrane either in complex or separately. TbpB has high affinity for iron-bound transferrin (Renauld-Mongenie et al. 1998; Retzer et al. 1998), and is predicted to be bilobal (like hTF) (Fuller et al. 1996), though how TbpB interacts with hTF and with TbpA is currently unclear. It appears that the N-lobe of $\mathrm{TbpB}$ interacts with both TbpA and with the C-lobe of hTF, and that there might be a secondary site on the C-lobe of TbpB that interacts with the N-lobe of hTF (Boulton et al. 1999). How TbpB discriminates between the states of hTF (iron-free, iron bound to the $\mathrm{N}$-lobe, iron bound to the $\mathrm{C}$-lobe or iron 
bound in both lobes) is also currently unknown, though it likely involves an interaction with multiple subdomains, since the structure of the subdomains (at least for the N-lobe) and the relative orientations of the lobes of hTF does not appear to change as iron is bound or released (Jeffrey et al. 1998; MacGillivray et al. 1998; Wally et al. 2006).

It is thought that iron-bound hTF is initially captured by $\mathrm{TbpB}$, and is thereby brought into proximity to bind to TbpA. This hTF-TbpATbpB complex is presumed to be the functional unit for iron removal and import into the bacterial periplasm. Iron is removed from the hTF lobe(s) by an unknown mechanism. This action requires that the hTF iron-containing lobe(s) must be opened to disrupt the strongly coordinated iron. This opening could result from separation of the subdomains or from a simple twisting action. However, the movement must be performed in a way that maintains a protected environment for the iron to move through the TbpA barrel and not be lost to the extracellular environment. If $\mathrm{TbpA}$ is structurally similar to other TonB-dependent iron transporters (Ferguson et al. 1998, 2002; Locher et al. 1998; Buchanan et al. 1999; Chimento et al. 2003; Kurisu et al. 2003; Yue et al. 2003; Cobessi et al. 2005a, b), the plug domain would be positioned inside the $\beta$-barrel, extending from the periplasm (where the TonB box resides) to the extracellular surface. In all known TonB-dependent transporter structures, the floor of the substrate binding pocket is formed by the apical loops of the plug domain, and residues in the plug domain directly coordinate the bound substrate. It is possible therefore that TbpA plug residues provide coordination for iron when it is extracted from hTF. When TonB interacts with the hTF-TbpA-TbpB complex, iron is released from hTF and transported into the periplasm. The transport process may involve partial or complete extraction of the plug domain from the barrel, but the precise mechanism is currently unknown. Once iron is removed from hTF, TbpB will no longer bind it and this might cause dissociation of the complex. However, TbpA binds both iron-free and iron-bound hTF (Krell et al. 2003); how the iron-free hTF is eventually released from TbpA is unknown.
Figure 6 shows predicted topology diagrams of TbpA and LbpA from Neisseria meningitidis. Although these proteins interact with host ironcontaining proteins that share great structural and sequence homology, their proposed topologies are quite different. In both topology diagrams, TbpA and LbpA contain large extracellular loops that might facilitate the interaction between the bacterial receptors and the relatively large host proteins. However, the distribution and nature of the loops are predicted to differ greatly in the two bacterial proteins. Furthermore, the putative size of the plug domain in the two receptors is different. The plug domain is predicted to be larger in TbpA as compared to LbpA, though other predictions for TbpA have a similarly sized plug (Oakhill et al. 2005). Having a larger plug domain might facilitate more interactions between the plug and the hTF, allowing direct transfer of the iron to the plug. The topology predictions suggest that the structure of TbpA (and possibly LbpA) might differ somewhat from other TonB-dependent iron transporters, as is suggested by their much larger size (approximately $20 \mathrm{kDa}$ larger than most TonB-dependent transporters). Since the bacteria make two different transport complexes for hTF and LTF, this suggests that the binding and iron-removal requirements differ for the two host iron-containing proteins. It is likely that the same features which affect iron affinity and TFR discrimination between hTF and LTF, may also contribute to the need for independent bacterial receptors.

\section{Conclusions}

Comparison of the structures of iron-free hTF and LTF has revealed several distinctions that could be important in the differing iron and receptor binding properties of these two proteins. Though hTF and LTF are overall quite similar in sequence and structure, they differ in the structure of their inter-lobe linker (helical in LTF and unstructured in hTF), the presence of a salt bridge between the helical linker of LTF and its C-lobe which is absent in hTF, their pattern of disulfide bonding (inter-subdomain bonding in hTF but not in LTF), the relative 




ADEHARIEGARRGTHILLAEAGGIRGALAI SQTLGRKGSYAT KAVEISKSNSVEQGSGNLAGSVAFCTKTADDVIGEGRQWGIQS $\mathrm{V}$

NEYEIENIAGSSGATRTGGLAAQATYSQIQSVGDVTLSVRNKD

VKSSDTLSKEOVTNTRDTRRYPPGTAVYROGRGASSGYSSTRG

L

KGLGTVENDRRTKQKKUKVQITDLQKRQAQGAQVNE

ThpA

Fig. 6 Topology models of TbpA (left) and LbpA (right) from Neisseria meningitidis strain MC58 (Tettelin et al. 2000). The plug domains and periplasmic loops are highlighted in yellow, the $\beta$-strands are highlighted in turquoise and the extracellular loops in light grey. Cysteine
QRAKLGAGF SKDGWEGLLIRTERQGRETRPHG

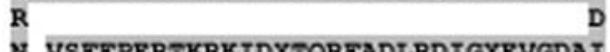

F

K E

S $G$

G $\mathrm{E}$

Y R

A $\mathbf{E}$

LLGKGKPEVVEGSVC NSGILFKPNRHFSV A

$\mathrm{G}$ W

KDDQNNEDFGTSE Y S IGFLEQTSPTRFGS

s

K $\mathrm{K}$

KSCRASADK I $\begin{array}{llll} & \text { E } & \text { D } & \text { I }\end{array}$

D $P$ R V V Y

VAPYVA Y A L Y H

A $\mathbf{P}$

Q $V$ TG

C $S$ A R R D

I A Y G YGTDA $\bar{N} Y N S G Y$

KPKSV

G K A G Y K

L D $\mathrm{B}$ R $\mathrm{S}$

W L \& R \& KDRE

S A A I K S

K G S L E G

K S S K R K

I S RY

RWY N K S

A R Q

L $P$ D K RHG

I G G R G

I $\mathrm{L}$ A $\mathrm{A}$ A $\mathrm{D}$

YLPEV NRE PEQAKGRA S E Y

DGNDMY NEPRERVLESLW A G

$S K E$ P R D D H R K PALP Y E S T R I $Y$ H I G I Y I I A I P L A D $=0 Y$ L L R D D D ID A R IN I L N K L P A R A D N A Y Y D F E V E A G F K D Q G D N D L A

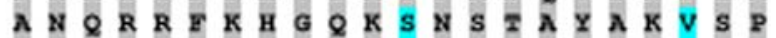
A Q A S Q R I B Q B N K E Y G V V S E G E L E G K D R R K N A I R K R Q G Q K A V R K N R S I G S D V D H D N L N P S Y N N TRVWDL Y R E L Y I A R T S L V F R W Q L Y KRSNGSR N F D R IN S S FV A A F L L L A F L G R I I Y L L R L A K E K V V G G S G N E S D K V K Y A V E E L G P I L A F L D L I I I G L I K N L A R E G G N G L K E I R S G E R L G N R G E G I A X V I R X D K L E ELL D I E A L A K G E S R Y E V R S B S D P Y N G G IN K L Q L F H E Y W L H I F W G Y Y W L Y E A NQP GGR W N E N K K GKY K E N G D K R I

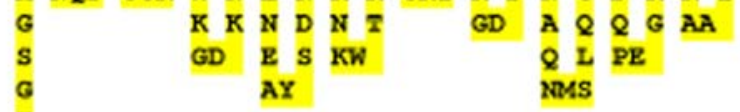

H

SSGAGKDIEVISINEYEIENIAGSGGRGGYGSLSGQVIFAQIQ LGIRDLTRYDPGVAVVEQGNGASGGYSIRGVDKRRVAVSVGV $\mathrm{V}$

QEKOILESTKVIKGLGTAEKSRRGVKAARVIIEKLSQTQAADP

AQAGGA

Ibpa

residues are underlined. The TonB boxes of the proteins are shown in green. The models were created using the PRED-TMBB software (http://bioinformatics.biol.uoa.gr/ PRED-TMBB/input.jsp) (Bagos et al. 2004) 
orientation of their lobes to one another (the C-lobe of LTF is rotated closer to the N-lobe as compared to hTF), the dilysine trigger and triad residues in hTF which are not present in LTF, the openness of their C-lobes (being more open in hTF), the structure of the C-lobe hinge regions (unstructured in hTF and $\beta$-strands in LTF), and their inter-lobe interactions (salt bridge between the C-terminal helix and N-lobe of hTF which is not found in LTF). Analysis of these differences increases our understanding of the divergent functions of these two proteins, as well the necessity for pathogenic bacteria to express independent receptors for two such similar proteins. Crystal structures of the bacterial receptors in complex with their host substrates should provide further insight into these interactions.

Acknowledgments We thank Tim Fritz (NIDDK/NIH) for critically reading the manuscript. This work is supported by the Intramural Research Program of the $\mathrm{NIH}$, National Institute of Diabetes and Digestive and Kidney Diseases.

\section{References}

Aisen P, Leibman A, Zweier J (1978) Stoichiometric and site characteristics of the binding of iron to human transferrin. J Biol Chem 253:1930-1937

Anderson BF, Baker HM, Norris GE, Rice DW, Baker EN (1989) Structure of human lactoferrin: crystallographic structure analysis and refinement at $2.8 \AA$ resolution. J Mol Biol 209:711-734

Anderson JE, Sparling PF, Cornelissen CN (1994) Gonococcal transferrin-binding protein 2 facilitates but is not essential for transferrin utilization. J Bacteriol 176:3162-3170

Bagos PG, Liakopoulos TD, Spyropoulos IC, Hamodrakas SJ (2004) PRED-TMBB: a web server for predicting the topology of beta-barrel outer membrane proteins. Nucleic Acids Res 32:W400-W404

Baker HM, Baker EN (2004) Lactoferrin and iron: structural and dynamic aspects of binding and release. Biometals 17:209-216

Beatty EJ, Cox MC, Frenkiel TA et al (1996) Interlobe communication in 13C-methionine-labeled human transferrin. Biochemistry 35:7635-7642

Boulton IC, Gorringe AR, Carr RJ et al (1997) Characterisation of the meningococcal transferrin binding protein complex by photon correlation spectroscopy. FEBS Lett 414:409-413

Boulton IC, Gorringe AR, Allison N et al (1998) Transferrin-binding protein B isolated from Neisseria men- ingitidis discriminates between apo and diferric human transferrin. Biochem J 334:269-273

Boulton IC, Gorringe AR, Shergill JK, Joannou CL, Evans RW (1999) A dynamic model of the meningococcal transferrin receptor. J Theor Biol 198:497-505

Boulton IC, Yost MK, Anderson JE, Cornelissen CN (2000) Identification of discrete domains within gonococcal transferrin-binding protein A that are necessary for ligand binding and iron uptake functions. Infect Immun 68:6988-6996

Buchanan SK, Smith BS, Venkatramani L et al (1999) Crystal structure of the outer membrane active transporter FepA from Escherichia coli. Nat Struct Biol 6:56-63

Cheng Y, Zak O, Aisen P, Harrison SC, Walz T (2004) Structure of the human transferrin receptor-transferrin complex. Cell 116:565-576

Cheng Y, Zak O, Aisen P, Harrison SC, Walz T (2005) Single particle reconstruction of the human apotransferrin-transferrin receptor complex. J Struct Biol 152:204-210

Chimento DP, Mohanty AK, Kadner RJ, Wiener MC (2003) Substrate-induced transmembrane signaling in the cobalamin transporter BtuB. Nat Struct Biol 10:394-401

Cobessi D, Celia H, Folschweiller N et al (2005a) The crystal structure of the pyoverdine outer membrane receptor FpvA from Pseudomonas aeruginosa at 3.6 angstroms resolution. J Mol Biol 347:121-134

Cobessi D, Celia H, Pattus F (2005b) Crystal structure at high resolution of ferric-pyochelin and its membrane receptor FptA from Pseudomonas aeruginosa. J Mol Biol 352:893-904

Cornelissen CN, Biswas GD, Tsai J et al (1992) Gonococcal transferrin-binding protein 1 is required for transferrin utilization and is homologous to TonBdependent outer membrane receptors. J Bacteriol 174:5788-5797

Day CL, Stowell KM, Baker EN, Tweedie JW (1992) Studies of the N-terminal half of human lactoferrin produced from the cloned cDNA demonstrate that interlobe interactions modulate iron release. J Biol Chem 267:13857-13862

DeLano WL (2002) The PyMOL molecular graphics system. DeLano Scientific, San Carlos

Evans RW, Oakhill JS (2002) Transferrin-mediated iron acquisition by pathogenic Neisseria. Biochem Soc Trans 30:705-707

Ferguson AD, Hofmann E, Coulton JW, Diederichs K, Welte W (1998) Siderophore-mediated iron transport: crystal structure of FhuA with bound lipopolysaccharide. Science 282:2215-2220

Ferguson AD, Chakraborty R, Smith BS et al (2002) Structural basis of gating by the outer membrane transporter FecA. Science 295:1715-1719

Fleming MD, Romano MA, Su MA et al (1998) Nramp2 is mutated in the anemic Belgrade (b) rat: evidence of a role for Nramp2 in endosomal iron transport. Proc Natl Acad Sci USA 95:1148-1153

Fuller CA, Retzer MD, Jacobs E, Schryvers AB (1996) Evidence for a bilobed structure for meningcoccal 
transferrin receptor binding protein B. In: Proceedings of the 10th international pathogenic Neisseria conference, Baltimore, pp 72-73

Giannetti AM, Snow PM, Zak O, Bjorkman PJ (2003) Mechanism for multiple ligand recognition by the human transferrin receptor. PLoS Biol 1:E51

Giannetti AM, Halbrooks PJ, Mason AB et al (2005) The molecular mechanism for receptor-stimulated iron release from the plasma iron transport protein transferrin. Structure 13:1613-1623

Guex N, Peitsch MC (1997) SWISS-MODEL and the Swiss-PdbViewer: an environment for comparative protein modeling. Electrophoresis 18:2714-2723

Guha Thakurta P, Choudhury D, Dasgupta R, Dattagupta JK (2003) Structure of diferric hen serum transferrin at $2.8 \AA$ resolution. Acta Crystallogr D Biol Crystallogr 59:1773-1781

Gumerov DR, Mason AB, Kaltashov IA (2003) Interlobe communication in human serum transferrin: metal binding and conformational dynamics investigated by electrospray ionization mass spectrometry. Biochemistry 42:5421-5428

Halbrooks PJ, He QY, Briggs SK et al (2003) Investigation of the mechanism of iron release from the C-lobe of human serum transferrin: mutational analysis of the role of a $\mathrm{pH}$ sensitive triad. Biochemistry 42:37013707

Hall DR, Hadden JM, Leonard GA et al (2002) The crystal and molecular structures of diferric porcine and rabbit serum transferrins at resolutions of 2.15 and $2.60 \AA$ A, respectively. Acta Crystallogr D Biol Crystallogr 58:70-80

Harris DC, Aisen P (1989) Iron carriers and iron proteins. VCH Publishers, New York

He QY, Mason AB, Tam BM, MacGillivray RT, Woodworth RC (1999) Dual role of Lys206-Lys296 interaction in human transferrin N-lobe: iron-release trigger and anion-binding site. Biochemistry 38:9704-9711

Jameson GB, Anderson BF, Norris GE, Thomas DH, Baker EN (1999) Structure of human apolactoferrin at 2.0 A resolution. Refinement and analysis of ligandinduced conformational change. Addendum. Acta Crystallogr D Biol Crystallogr 55:1108

Jeffrey PD, Bewley MC, MacGillivray RT et al (1998) Ligand-induced conformational change in transferrins: crystal structure of the open form of the $\mathrm{N}$ terminal half-molecule of human transferrin. Biochemistry 37:13978-13986

Khan JA, Kumar P, Paramasivam M et al (2001) Camel lactoferrin, a transferrin-cum-lactoferrin: crystal structure of camel apolactoferrin at $2.6 \AA$ resolution and structural basis of its dual role. J Mol Biol 309:751-761

Krell T, Renauld-Mongenie G, Nicolai MC et al (2003) Insight into the structure and function of the transferrin receptor from Neisseria meningitidis using microcalorimetric techniques. J Biol Chem 278:14712-14722

Kumar P, Khan JA, Yadav S, Singh TP (2002) Crystal structure of equine apolactoferrin at $303 \mathrm{~K}$ providing further evidence of closed conformations of $\mathrm{N}$ and $\mathrm{C}$ lobes. Acta Crystallogr D Biol Crystallogr 58:225-232
Kurisu G, Zakharov SD, Zhalnina MV et al (2003) The structure of BtuB with bound colicin E3 R-domain implies a translocon. Nat Struct Biol 10:948-954

Kurokawa H, Mikami B, Hirose M (1994) Crucial role of intralobe peptide-peptide interactions in the uptake and release of iron by ovotransferrin. J Biol Chem 269:6671-6676

Kurokawa H, Mikami B, Hirose M (1995) Crystal structure of diferric hen ovotransferrin at $2.4 \AA$ resolution. J Mol Biol 254:196-207

Kurokawa H, Dewan JC, Mikami B, Sacchettini JC, Hirose M (1999) Crystal structure of hen apo-ovotransferrin. Both lobes adopt an open conformation upon loss of iron. J Biol Chem 274:28445-28452

Lawrence CM, Ray S, Babyonyshev M et al (1999) Crystal structure of the ectodomain of human transferrin receptor. Science 286:779-782

Lin LN, Mason AB, Woodworth RC, Brandts JF (1991) Calorimetric studies of the binding of ferric ions to ovotransferrin and interactions between binding sites. Biochemistry 30:11660-11669

Lin LN, Mason AB, Woodworth RC, Brandts JF (1994) Calorimetric studies of serum transferrin and ovotransferrin. Estimates of domain interactions, and study of the kinetic complexities of ferric ion binding. Biochemistry 33:1881-1888

Liu R, Guan JQ, Zak O, Aisen P, Chance MR (2003) Structural reorganization of the transferrin C-lobe and transferrin receptor upon complex formation: the C-lobe binds to the receptor helical domain. Biochemistry 42:12447-12454

Locher KP, Rees B, Koebnik R et al (1998) Transmembrane signaling across the ligand-gated FhuA receptor: crystal structures of free and ferrichrome-bound states reveal allosteric changes. Cell 95:771-778

MacGillivray RT, Moore SA, Chen J et al (1998) Two high-resolution crystal structures of the recombinant N-lobe of human transferrin reveal a structural change implicated in iron release. Biochemistry 37:7919-7928

Mazurier J, Spik G (1980) Comparative study of the ironbinding properties of human transferrins. I. Complete and sequential iron saturation and desaturation of the lactotransferrin. Biochim Biophys Acta 629:399-408

Moore SA, Anderson BF, Groom CR, Haridas M, Baker EN (1997) Three-dimensional structure of diferric bovine lactoferrin at $2.8 \AA$ resolution. J Mol Biol 274:222-236

Nurizzo D, Baker HM, He QY et al (2001) Crystal structures and iron release properties of mutants (K206A and K296A) that abolish the dilysine interaction in the N-lobe of human transferrin. Biochemistry 40:1616-1623

Oakhill JS, Sutton BJ, Gorringe AR, Evans RW (2005) Homology modelling of transferrin-binding protein A from Neisseria meningitidis. Protein Eng Des Sel 18:221-228

Okamoto I, Mizutani K, Hirose M (2004) Iron-binding process in the amino- and carboxyl-terminal lobes of ovotransferrin: quantitative studies utilizing single Fe3(-binding mutants. Biochemistry 43:11118-11125 
Pawelek PD, Croteau N, Ng-Thow-Hing C et al (2006) Structure of TonB in complex with FhuA, E. coli outer membrane receptor. Science 312:1339-1402

Peterson NA, Arcus VL, Anderson BF et al (2002) "Dilysine trigger" in transferrins probed by mutagenesis of lactoferrin: crystal structures of the R210G, R210E, and R210L mutants of human lactoferrin. Biochemistry 41:14167-14175

Rawas A, Muirhead H, Williams J (1996) Structure of diferric duck ovotransferrin at $2.35 \AA$ resolution. Acta Crystallogr D Biol Crystallogr 52:631-640

Rawas A, Muirhead H, Williams J (1997) Structure of apo duck ovotransferrin: the structures of the $\mathrm{N}$ and $\mathrm{C}$ lobes are in the open form. Acta Crystallogr D Biol Crystallogr 53:464-468

Renauld-Mongenie G, Latour M, Poncet D, Naville S, Quentin-Millet MJ (1998) Both the full-length and the $\mathrm{N}$-terminal domain of the meningococcal transferrinbinding protein $\mathrm{B}$ discriminate between human iron-loaded and apo-transferrin. FEMS Microbiol Lett 169:171-177

Retzer MD, Yu R, Zhang Y, Gonzalez GC, Schryvers AB (1998) Discrimination between apo and iron-loaded forms of transferrin by transferrin binding protein B and its N-terminal subfragment. Microb Pathog 25:175-180

Rohde KH, Dyer DW (2003) Mechanisms of iron acquisition by the human pathogens Neisseria meningitidis and Neisseria gonorrhoeae. Front Biosci 8:d1186d1218

Shultis DD, Purdy MD, Banchs CN, Wiener MC (2006) Outer membrane active transport: structure of the BtuB:TonB complex. Science 312:1396-1399

Teh M, Hewitt J, Ung KC et al (2005) Identification of the epitope of a monoclonal antibody that disrupts binding of human transferrin to the human transferrin receptor. FEBS J 272:6344-6353

Tettelin H, Saunders NJ, Heidelberg J et al (2000) Complete genome sequence of Neisseria meningitidis serogroup B strain MC58. Science 287:1809-1815
Thakurta PG, Choudhury D, Dasgupta R, Dattagupta JK (2004) Tertiary structural changes associated with iron binding and release in hen serum transferrin: a crystallographic and spectroscopic study. Biochem Biophys Res Commun 316:1124-1131

Thompson JD, Higgins DG, Gibson TJ (1994) ClustalW: improving the sensitivity of progressive multiple sequence alignment through sequence weighting, position-specific gap penalties and weight matrix choice. Nucleic Acids Res 22:4673-4680

Wally J, Halbrooks PJ, Vonrhein C et al (2006) The crystal structure of iron-free human serum transferrin provides insight into inter-lobe communication and receptor binding. J Biol Chem 281:24934-24944

Wandersman C, Delepelaire P (2004) Bacterial iron sources: from siderophores to hemophores. Annu Rev Microbiol 58:611-647

Ward PP, Zhou X, Conneely OM (1996) Cooperative interactions between the amino- and carboxyl-terminal lobes contribute to the unique iron-binding stability of lactoferrin. J Biol Chem 271:12790-12794

Wiener M (2005) TonB-dependent outer membrane transport: going for Baroque? Curr Opin Struct Biol 15:394-400

Woodbury RG, Brown JP, Yeh MY, Hellstrom I, Hellstrom KE (1980) Identification of a cell surface protein, p97, in human melanomas and certain other neoplasms. Proc Natl Acad Sci USA 77:2183-2187

Xu G, Liu R, Zak O, Aisen P, Chance MR (2005) Structural allostery and binding of the transferrin $\times$ receptor complex. Mol Cell Proteomics 4:1959-1967

Yue WW, Grizot S, Buchanan SK (2003) Structural evidence for iron-free citrate and ferric citrate binding to the TonB-dependent outer membrane transporter FecA. J Mol Biol 332:353-368 